\title{
Dimensions of Quality and Their Increasing Relevance for Visceral Medicine in Germany
}

\author{
Wolfram Keßler ${ }^{a} \quad$ Claus-Dieter Heidecke ${ }^{a, b}$ \\ a Department of General, Visceral, Thoracic and Vascular Surgery, Universitätsmedizin Greifswald, Greifswald, Germany; \\ ${ }^{\text {b } C h i r u r g i s c h e ~ A r b e i t s g e m e i n s c h a f t ~ f u ̈ r ~ Q u a l i t a ̈ t, ~ S i c h e r h e i t ~ u n d ~ V e r s o r g u n g s f o r s c h u n g ~(C A O S-V), ~ D e u t s c h e ~ G e s e l l s c h a f t ~ f u ̈ r ~ C h i r u r g i e ~}$ \\ (DGCH), Berlin, Germany
}

\section{Keywords}

Quality · Quality indicators · Registries .

Administrative data

\section{Summary}

Background: 'Quality in medicine' is a term used in a broad sense. In this work the definition and dimensions of quality in medicine and the implementation of a measurement and reporting system in Germany are discussed. Existing applications are described and possible future effects are pointed out. Methods: The ongoing process of implementing a quality reporting system into the German healthcare system is studied by publicly available legal texts, published reactions of stakeholders and publications of G-BA and IQTIG. Definitions of quality, dimensions of quality and quality measurement in medicine are studied by using textbooks as well as the world wide web and PubMed search. Results: Donabedian's 'dimensions of quality' are fundamental in dealing with quality in medicine. Existing measurement and reporting systems have immanent strengths and weaknesses, as the definition of quality is affected by one's point of view. The legislator will have to decide which 'dimension of quality' is mandatory and how to measure it. Conclusion: Quality has become a control instrument with unforeseeable consequences. A clear definition of the used quality concept is as essential as the use of feasible measurement and reporting systems. The use of routine data could be an interesting option.

(C) 2017 S. Karger GmbH, Freiburg

\section{Introduction}

The German lawgiver has set new rules in 2016 by implementing the so-called 'hospital structure law' [1]: 'the quality of inpatient medical care in the future will be taking on a larger role accompanied by stricter controlling as well as consequential improvement. Quality will be introduced as a criterion in hospital planning and obligation to the quality assurance regulations developed by the Federal Joint Committee (G-BA) will be strengthened. The minimum quantity regulation will be applied in strict legal accordance to the law ... Autonomy for medical decisions will become increased ... Hospital compensation will become closely tied to qualitative aspects and hospital quality reports will be designed in a more patient-friendly format so that useful information is more easily available.' As can be seen from this, the legislator is serious about enforcing quality measures.

Quality has always been an important aspect in surgery. Theodor Billroth's statement 'Nothing is worse than the obscurity of one's own actions' already addressed this long ago. A critical view of one's own work is essential. The quality offensive undertaken by the German Federal Government also focuses on the high value of quality. Proof of quality (a monodimensional term generally used by politics) is, however, clearly more problematic. The primary issue is that the term quality is extremely difficult to define.

\section{The Problem with the Term 'Quality'}

On the homepage of the German Society for Quality (Deutsche Gesellschaft für Qualität e.V.) the following definition of quality can be found: 'To do the right thing correctly' [2]. Thus, it can already be assumed that quality is based upon whoever is judging it or, in other words, whoever rules what is correct or sets the requirements to develop a precedent.

\section{KARGER}

(๑) 2017 S. Karger GmbH, Freiburg

Fax +497614520714
Prof. Dr. med. Claus-Dieter Heidecke, MBA

Klinik \& Poliklinik für Allgemeine Chirurgie, Viszeral-, Thorax- und Gefäßchirurgie Universitätsmedizin Greifswald

Ferdinand-Sauerbruch-Straße, 17475 Greifswald, Germany

heidecke@ uni-greifswald.de 
Two differing quality concepts can be derived from classic business management teachings. The first covers product-based quality terms which can be developed and measured according to tangible characteristics of products. The second involves customer-based quality terms, whereby the extent of quality reflects its usefulness or the value of a product observed by the consumer [3]. Ultimately, quality will be evaluated by the comparison between the usefulness and the price/sacrifice by the customer. Surgeons can certainly identify with this in terms of medical products for the operating room. In the same sense, it can be concluded that for a health system, quality criteria compliance and its set cost would have to be defined beforehand.

A successful definition of quality in medicine was offered by the Institute of Medicine in 1990: Quality of care is the degree to which health services for individuals and populations increase the likelihood of desired health outcomes and are consistent with current professional knowledge [4]. This definition describes the quality of health provision from the viewpoint of the patient and correlates it with the status of research or evidence-based medicine. Though more theoretical than actually realizable, this definition gave cause for discussion not only in the USA, pertaining to adjustments of health care policy to the health care system. In 2001, the ' 6 domains of health care quality' were presented $[5,6]$. The quality of the health care system should hereby fulfill the six aspects which are stated in table 1.

The distribution into six categories provides the possibility of working structurally within each dimension, whereby distinct goals for each aspect should be provided. In Germany, general regulations and particularly legislation are based upon the classical $d i-$ mensions of quality first published by Donabedian [7] in 1966. He divided quality within health care into three categories: structural, process and outcome quality. According to Donabedian, structural quality is based upon the right prerequisites in facility, equipment as well as human and technical resource. Process quality is simply doing the right thing correctly by delivering proper treatment. Outcome quality is the accomplishment of an intended condition [7]. Often a fourth element is introduced involving the so-called 'quality of experience', where patient satisfaction, in the widest sense, is strongly focused upon in terms of participation and less in terms of wellness [8].

Though structure and process quality can be easily measured and therefore quickly optimized, there is no guarantee that the outcome quality will simultaneously increase, too. Exclusive focus solely upon structure and process measures only makes sense when a positive correlation to outcome quality can be all but proven or substantiated [9].

\section{How Is Quality Measured in Medicine?}

The definition of quality is not simple, while its detailed measurement is similarly complex. Quite often quality indicators (quantitative figures) are used in several areas of the health care system to evaluate presumably important functions. An indicator, how-
Table 1. Factors for assessing the quality of the health care system

\begin{tabular}{ll}
\hline Safe & $\begin{array}{l}\text { Avoiding harm to patients from the care that is intended } \\
\text { to help them. }\end{array}$ \\
\hline Effective & $\begin{array}{l}\text { Providing services based on scientific knowledge to all } \\
\text { who could benefit and refraining from providing services to } \\
\text { those not likely to benefit (avoiding underuse and misuse) }\end{array}$ \\
\hline Patient-centered & $\begin{array}{l}\text { Providing care that is respectful of and responsive to } \\
\text { individual patient preferences, needs, and values and } \\
\text { ensuring that patient values guide all clinical decisions. }\end{array}$ \\
\hline Timely & $\begin{array}{l}\text { Reducing waiting time and sometimes harmful delays for } \\
\text { both those who receive and those who give care. }\end{array}$ \\
\hline Efficient & $\begin{array}{l}\text { Avoiding waste, including waste of equipment, supplies, } \\
\text { ideas, and energy. }\end{array}$ \\
\hline Equitable & $\begin{array}{l}\text { Providing care that does not vary in quality because } \\
\text { of personal characteristics such as gender, ethnicity, } \\
\text { geographic location, and socioeconomic status. }\end{array}$ \\
\hline
\end{tabular}

ever, is not a direct measurement of quality. It is more a tool which can be used in performance evaluation which directs attention to potential problem areas requiring intensive examination within an organization. The evaluation of quality via the use of indicators initially serves the improvement of quality [10]. Commonly, well-defined structure, process or outcome indicators serve as quality indicators in medicine. The question of which quality indicator will be drawn upon for which question is up to the institution which sets the standards or the agency which operates registries. Controversies do naturally arise, as was recently the case concerning the first quality report of the Institute for Quality Assurance and Transparency in Healthcare (Institut für Qualitätssicherung und Transparenz im Gesundheitswesen (IQTIG)). Similarly, the quality indicators obtained from routine data employed by the health insurances, namely the AOK (local health care funds) together with the WIdO Institute (Wissenschaftliches Institut der AOK; research institute of the local health care funds), are the subject of extensive discussion. Here, ICD (International Classification of Diseases) codes or OPS (Operation and Procedure codes) for certain illnesses are used as quality indicators. In terms of risk adjustment, it is important to include the patients' severity of disease in the quality evaluation. It is also essential that the quality indicators include correct complication potential for an index patient which, unfortunately, the German ICD-10 system does not always satisfactorily provide (e.g. classification of liver cirrhosis, intake of corticosteroids or immunosuppressives).

\section{Prospects for Quality Rating by Internal Quality Assurance}

In the past, quality was documented for self-interest reasons in more or less sophisticated databases for the purpose of internal quality assurance. Instruments such as the Clavien-Dindo Classifi- 
cation [11] were often employed to evaluate quality outcome in patients with abnormal courses of a disease.

Quality assurance within a 'protected area' has been provided by medical societies or the An-Institute for Quality Assurance in Operative Medicine in Magdeburg, thereby allowing benchmarking with other hospitals. In the past few years, medical societies such as the German Society for General and Visceral Surgery (DGAV) and the German Society of Orthopedic and Trauma Surgery (DGOU) have gained recognition for the development of such registries. It can be criticized, however, that the definition of the parameters for quality indicators are chosen arbitrarily by the registry which could make the fulfillment of requirements easy or difficult. The administrators of the registry determine the 'rules of the game' for which and to which extent parameters are to be documented. Critics claim that overwhelming requirements of clinical parameters would lead to incomplete data reporting, thereby lowering the quality. Well-organized registry data are, however, a suitable option for quality assurance in terms of providing realistic answers to certain care provider questions $[12,13]$.

A further form of internal quality assurance with the option of benchmark creation is possible by means of certification by e.g. the DGAV or the German Cancer Society (DKG). Within the certification framework of the DGAV, participation in the registry system (StuDoQ) is mandatory. Outcome and process quality are closely monitored at the time of certification as well as at re-certification [14]. All centers must fulfill the minimum quantity regulations. Since 2013, quality assurances for the DKG and the German Cancer Aid (DKH) have been harmonized [15]. Both organizations distribute questionnaire forms. The goal is that the same rules apply for oncological treatment in all certified centers, thus resulting in comparable treatment results. In order to form quality indicators from guidelines, a methodology paper was developed using the framework of the oncological guideline programs from the DKG, the DKH, and the Association of the Scientific Medical Societies in Germany (AWMF) [16]. Revision of the oncological quality indicators is undertaken yearly. All dimensions of quality for the certified oncological sector are questioned, starting with the qualification of the provider (structure quality), the availability of distinct treatment process elements (process quality), patient follow-up controls (outcome quality), and, finally, critical self-assessment with problem cases (morbidity-mortality conferences). Patient satisfaction is also taken into consideration by means of patient questionnaires.

\section{Feasibility of Quality Assessment by External Quality Assurance}

External quality assurance is long well established in Germany. In 2016, this function was transferred to the newly founded IQTIG as cross-sectoral quality assurance. According to the data of the external Quality and Safety (QS) from 2008 until 2011, the quality of inpatient care had either improved (36\%) or remained stable (59\%). Only $5 \%$ of the quality indicators had worsened [17].
The quality offensive in medicine undertaken by the Federal Government was effectively implemented via the so-called Hospital Structures Act and an announcement in the Federal Law Gazette. According to $\$ 135$ SGB V (social code book V), quality assurance in medicine is to be understood as widely comprehensive and is separated from quality management in the facility. Participation in both processes (cross-sectoral quality assurance as well as internal quality management of the facility) is compulsory and encompasses primarily outcome quality. All players in health care (contractual doctors, medical care centers, accredited hospitals, providers of preventive treatment or rehabilitation measures as well as facilities with patient care provision contracts according to $\$ 111 \mathrm{a})$ have to participate. In addition, according to $\$ 136 \mathrm{SGB} \mathrm{V}$, minimal requirements regarding structure, process and outcome quality should be defined by the Federal Joint Committee (Gemeinsamer Bundesausschuss (G-BA)) in certain areas of medical care.

According to $\$ 137$ SGB V, within the framework of external comparative quality assurance for inpatient care, hospitals are obliged to report quality-relevant data for defined medical treatment areas to the IQTIG. Founded in 2015, the IQTIG presently includes 110 staff members and bears the entire responsibility for the legally determined quality assurance according to paragraphs 136ff und 137a SGB V since January 2016. An initial report has already been presented including 1,834 participating hospitals, 3.2 million quality assurance data records, 25 areas of treatment (QS procedure), 351 quality indicators, and 91 risk adjustment indicators [18]. As already mentioned, the quality indicators remain the subject of discussion, and appropriate further development remains the core task of the institute. An initial guideline for future hospital development planning according to relevant quality indicators in gynecology has been developed and was released on December 15th, 2016 [19].

Data delivered to the IQTIG are statistically evaluated according to nationwide, uniform concepts according to which risk adjustment takes place. The results are made available to the participating hospitals and are also made public in a comprehensible form [18]. Detection of site-related abnormalities is handled by structured dialogue pertaining to the regulations. In the instance of violations against quality requirements, the following measures of sanction according to $\$ 137$ SGB V are possible:

- reductions in reimbursement,

- loss of reimbursement rights for services where the minimal standards according $\$ 136$ SGB V are not met,

- report of violation of a third party,

- institution-related disclosure of information pertaining to nonadherence to quality requirements.

Furthermore, when authorized by the G-BA or defined posts, the Medical Service of the Statutory Health Insurance Funds (Medizinischer Dienst der Krankenkassen (MDK)) is permitted to make unannounced control checks of quality requirements, documentation of external quality assurance, and adherence to state quality regulations [20]. In addition, quality contracts with the health care insurances should also be deemed permissible [21]. 
Currently, the following medical areas from the operative specialties are presented:

- obstetrics,

- gynecological procedures (excluding hysterectomy),

- breast surgery,

- heart pacemaker provision including defibrillators,

- hip and knee endoprosthesis provision,

- carotid revascularisation,

- aortic valve and combined coronary and aortic valve surgery,

- transplantation surgery.

This list will naturally be expanded in the future.

The development of expanded monitoring as well as the option to sanction health care providers are also new since the founding of the IQTIG; these measures can and will very likely be employed in the planning of the hospital landscape.

\section{Feasibility of Quality Assurance Using Routine Data}

In 2002, quality assurance through routine data (Qualitätssicherung mit Routinedaten (QSR)) was initiated as a cooperative development project by the WIdO, the AOK Federal Association, the Helios Clinics Group, and the Research and Development Institute for Social and Public Health Saxony-Anhalt (Forschungsund Entwicklungsinstitut für das Sozial- und Gesundheitswesen Sachsen-Anhalt). The project has been continuously developed further by the WldO since 2008. The goal was to develop and establish a low-effort, non-complex quality measurement method for Germany, using so-called routine data which would be focused on outcome quality. At present, 18 treatment areas are covered by QSR indicators, two of which are visceral surgery-related: appendectomy and cholecystectomy. The treatment areas including benign thyroid disease have not been publicly reported. Treatment areas comprising gastroenterology or endoscopy do not exist yet.

QSR is based upon AOK billing data in an anonymous form (synonymous: routine data, administrative data, secondary data) which, according to $\$ 301$ SGB V, are reported to the health insurance by the hospitals. This data include information pertaining to the length of hospital stay, diagnoses, procedures, and amount of case-related revenues of the hospital for inpatient treatment. These data are combined with data (also made anonymous) regarding age and sex of the patient as well as insurance and survival status. Follow-up G-BA practice visits and readmissions are added to the data. Through the use of the QSR procedure it is ultimately possible to allocate various hospital stays and practice visits to one patient without repeated identification of the person [22].

The central advantage of QSR over traditional quality assurance processes is that occurrences such as ambulatory visits (not at the treating hospital) flow into the database and are included. In Germany, where the ambulatory and the inpatient sector are strictly separated, the QSR process offers a solution by providing an interface which, by means of cross-sectoral quality assurance, at best proves to be a challenge. Thus, a routine, long-term follow-up is possible for the first time. At the same time, additional documenta- tion can be avoided due to already available administrative and billing data being accessible. It can be readily assumed that the resource-relevant data submitted by the hospitals are complete because the hospital is steering its revenue by means of these data [22].

With the use of QSR, the quality measurement of hospitals is primarily directed toward analysis and evaluation of outcome indicators such as hospital mortality as well as 30-day, 90-day and 1 -year mortality rate. In addition, general as well as treatment-specific complications or adverse events during the hospital stay as well as complication-related readmission or undesired subsequent events occurring up to 1 year after the initial treatment are included [22]. A list of quality indicators for each medical area can be found in the indicator handbook [23].

The expected events are measured with the help of a logistic regression model. In order to guarantee a fair comparison between the hospitals, a risk adjustment according to patient characteristics is carried out. This risk adjustment is performed based upon sex, age, and approximately 30 relevant concomitant illnesses of the patients as well as other factors which are treatment-dependent, such as the employed procedures. Only concomitant illnesses which presumably existed prior to inpatient admission are used for risk adjustment. Risk adjustment is reviewed once a year and audited for systematic discrepancies. The QSR project is attended to by a scientific advisory board as well as by expert panels from the treatment areas (e.g. expert panel visceral surgery or endocrine surgery) [24].

Critics of QSR question whether risk adjustment can be correctly performed for individual high-risk patients or for hospitals with a high frequency of admitted high-risk patients (e.g. for the distribution of so-called 'life trees'). The problem therein is that sometimes the pre-existing morbidity of the patient cannot be clearly separated from newly acquired diagnoses due to the limited differentiation of the German ICD-10 system. As already mentioned, the rendering of specific patient conditions with the German ICD-10 system is insufficient. Therefore, a revision of the ICD-10 system would be necessary to make patient-related risk stratification more objective. There should also be better options to code the severity of manifestation of the main diagnosis. It is undeniable that in view of the complication potential, there is a considerable risk difference between a symptomatic cholecystolithiasis within a structurally intact gall bladder and a gangrenous cholecystitis in the presence of numerous inflammatory symptoms. If all cases of cholecystitis were exactly the same, then the point of time for surgical treatment would not have shown any difference for the patients' morbidity in the ACDC trial [25]. An ICD and OPS system which takes the need for quality assurance and risk stratification into consideration would clearly solve many problems.

\section{What Is Important for the Quality of Visceral Medicine in the Future?}

The current debate on quality encompasses all areas of medicine. Even if the vast majority of quality indicators is predominantly employed for operative procedures, it is only a question of 
time before the instruments for external quality assurance as well as those from the QSR will be applied for usage in the interventional and conservative treatment areas. It remains to be seen what the impact of the implementation of the Hospital Structures Act will be. In the presence of quality awards, surcharges, and strict minimum quantity regulations, there will definitely be 'winners and losers' within the system. The total number of monitored procedures/illnesses is still limited. Further extension of the QSR system in the future will not only have an impact on visceral surgery but also on gastroenterology and endoscopy. It can be expected that the reporting time intervals for QS data sent to the IQTIG will be distinctly shortened and that reporting will occur several times during the year [19].

It would be wise if the field of visceral medicine became involved in the debate on quality indicators as these data will eventually steer the health care system in the direction of 'pay for performance' (P4P). P4P basically means that health care providers should be motivated by differentiated financial incentives to deliver quality and efficiency in their code of practice in a sustainable, optimal and further developing manner. Until now, P4P has not demonstrated any significant benefits within the hospital sector [26]. Nevertheless, well-planned and orchestrated P4P studies should be performed before politics decides on new programs based upon unsatisfactory data [27]. Most importantly, new P4P instruments need to be found since those pre-existing indicators, which were not designed for $\mathrm{P} 4 \mathrm{P}$, should not be applied due to their unsuitability.

In the near future, escalated due to the minimal requirement regulation in the field of esophageal and pancreatic surgery, a stronger focus will be placed upon specialization. If this minimal requirement regulation is furthered by the legislator to other areas of surgery, a similar effect can be expected. Specialization and formation of specialized centers present an important prognosis factor for patients. Reports from countries where the centralization of complex surgical procedures has already taken place substantiate this fact [28]. A recent study commissioned by the Bertelsmann Foundation and presented by the Institute for Health and Social Research (Institut für Gesundheits- und Sozialforschung (IGES)) in Berlin confirmed these international findings. By means of such analyses, reliable knowledge can be gathered and used in the political discussion about health and the distribution of care [29]. The advantage of selected operations being frequently carried out at designated centers then appears economically sensible [30].
When it comes to quality assurance, several surgical societies have laid an excellent groundwork. The field of visceral surgery has done so due to the efforts of the DGAV. Numerous registries have been made available with the option of quality assurance as well as the opportunity to benchmark for participating centers. Some individual data sheets, however, are subjectively quite extensive which places a high demand on those colleagues responsible for the data input. Consequently, the completeness and validity of the entered data is determined by the institution itself. If the intention of the registry is a focus on health service research, outcome quality from registries may show results incongruent with the institution's quality expectations if the quality indicators were chosen as such.

Presently it is unclear in which direction the G-BA or the IQTIG wish to further develop the external quality assurance, i.e. related to procedures/operations or to diseases. It is also undecided how the IQTIG will value certificates and certification processes. Both decisions would set a trend as they would strengthen local or regional center development for oncological or complex visceral diseases such as obesity or inflammatory bowel disease. Together with a stricter minimum quantity regulation this would clearly change the hospital landscape. Furthermore, this would have an extensive impact upon the structure of departments as well as hospitals and thereby also upon residency programs.

The legislation during this period has developed instruments which could alter the health care system in a non-foreseeable manner. In addition to the current system, quality assurance using routine data will play a greater role as the health insurances actively promote it. The options of implementing 'quality contracts' [21] could provide allocative functions. The structural as well as process-related quality stipulations of the G-BA will regulate which institutions will be allowed to participate in providing health care. In addition, the emphasis on outcome quality should finally improve the delivery of medical care. It remains to be seen whether a concentration of the hospital landscape due to economic pressure will occur prior to the effects of these quality measures. In this case, not the 'best' hospitals will survive but the most efficient ones.

\section{Disclosure Statement}

WK and CDH have no conflicts of interest.

\section{References}

1 www.bundesgesundheitsministerium.de/themen/krankenversicherung/ krankenhausstrukturgesetz/khsg.html (last accessed: 4.1.2017)

2 www.dgq.de/service/faq/wissen (last accessed: 3.1.2017).

3 Vahlens Kompendium der Betriebswirtschaftslehre, Band 1, ed 5. München, Franz Vahlen, 2004, p 352.
4 Institute of Medicine; Committee to Design a Strategy for Quality Review and Assurance in Medicare; Lohr KN (ed): Medicare: A Strategy for Quality Assurance. Volume II: Sources and Methods. Washington, DC, Institute of Medicine, 1990. www.nap.edu.

5 www.ahrq.gov/professionals/quality-patient-safety/ talkingquality/create/sixdomains.html (last accessed: 22.12.2016).
6 Institute of Medicine (IOM): Crossing the Quality Chasm: A New Health System for the 21st Century. Washington, DC, National Academy Press, 2001.

7 Donabedian A: Evaluating the quality of medical care. Milbank Mem Fund Q 1966;44(suppl):166-206.

8 Gottschalk J: Das Unternehmen Krankenhaus zwischen Wettbewerbs- und Komplexitätsfalle. Berlin, epubli, 2014. 
9 Rubin HR, Pronovost P, Diette GB: The advantages and disadvantages of process-based measures of health care quality. Int J Qual Health Care 2001;13:469-474.

10 Kötter T, Schaefer F, Blozik E, Scherer M: Developing quality indicators: background, methods and problems (Article in German). Z Evid Fortbild Qual Gesundhwes 2011;105:7-12.

11 Dindo D, Demartines N, Clavien P-A: Classification of surgical complications: a new proposal with evaluation in a cohort of 6336 patients and results of a survey. Ann Surg 2004;240:205-213

12 Wellner UF, Lapshyn H, Bartsch DK, et al.; StuDoQ Pancreas study group and members of StuDoQ|Pancreas registry of the German Society for General and Visceral Surgery (DGAV): Laparoscopic versus open distal pancreatectomy - a propensity score-matched analysis from the German StuDoQ|Pancreas registry. Int J Colorectal Dis 2017;32:273-280.

13 Debus F, Lefering R, Frink M, et al: Numbers of severely injured patients in Germany. A retrospective analysis from the DGU (German Society for Trauma Surgery) Trauma Registry. Dtsch Arztebl Int 2015;112: 823-829.

14 www.dgav.de/fileadmin/media/texte_pdf/zertifizierung/ Zertifizierungsordnung_DGAV_5_1.pdf (last accessed: 7.1.2017)
15 www.krebsgesellschaft.de/deutsche-krebsgesellschaftwtrl/deutsche-krebsgesellschaft/presse/pressearchiv2013/ deutsche-krebsgesellschaft-und-deutsche-krebs.html (last accessed: 7.1.2017).

16 http://leitlinienprogramm-onkologie.de/uploads/tx_ sbdownloader/QIEP_OL_2013.pdf (last accessed: 7.1. 2017).

17 Ruckle F, Stausberg J: Trend of quality of hospital care in German hospitals between 2008 and 2011: a study from national quality assurance data (Article in German). Dtsch Med Wochenschr 2015;140:e36-42.

18 IQTIG: Qualitätsreport 2015. Berlin, Institut für Qualitätssicherung und Transparenz im Gesundheitswesen, 2016, pp 1-196. https://iqtig.org/downloads/ergebnisse/ qualitaetsreport/IQTIG-Qualitaetsreport-2015.pdf.

19 www.g-ba.de/downloads/39-261-2812/2016-12-15 PlanQI-RL_Erstfassung-PlanQI-RL.pdf (last accessed: 7.1.2017).

$20 § 275 a$, SGB V, Bundesgesetzblatt Jahrgang 2015, Teil I, Nr. 51. Bonn, 17.12.2015, p 2251.

$21 \$ 110 a$, SGB V, Bundesgesetzblatt Jahrgang 2015, Teil I, Nr. 51. Bonn, 17.12.2015, p 2244.

22 www.qualitaetssicherung-mit-routinedaten.de/imperial md/qsr/publikationen/wido_kra_qsr-abschlussbericht_ 0407.pdf (last accessed: 7.1.2017).

23 www.qualitaetssicherung-mit-routinedaten.de/imperia/ mdqsr/methoden/indikatorenhandbuch_mit_berichterstattung_2015.pdf (last accessed: 7.1.2017).
24 www.qualitaetssicherung-mit-routinedaten.de/entwicklung/experten/ (last accessed: 7.1.2017).

25 Gutt CN, Encke J, Köninger J, et al: Acute cholecystitis: early versus delayed cholecystectomy, a multicenter randomized trial (ACDC study, NCT00447304). Ann Surg 2013;258:385-393.

26 Milstein R, Schreyoegg J: Pay for performance in the inpatient sector: a review of $34 \mathrm{P} 4 \mathrm{P}$ programs in 14 OECD countries. Health Policy 2016;120:1125-1140.

27 Busse R: Pay-for-performance: time to act but also to provide further evidence. Health Policy 2016;120: 1123-1124.

28 de Wilde RF, Besselink MGH, van der Tweel I, et al: Impact of nationwide centralization of pancreaticoduodenectomy on hospital mortality. Br J Surg 2012;99: 404-410.

29 Nimptsch U, Peschke D, Mansky T: Minimum caseload requirements and in-hospital mortality: observational study using nationwide hospital discharge data from 2006 to 2013 (Article in German). Gesundheitswesen 2016;DOI: 10.1055/s-0042-100731.

30 von Dercks N, Gockel I, Mehdorn M, Lorenz D: Economic aspects of oncological esophageal surgery: centralization is essential (Article in German). Chirurg 2017;88:62-69. 